\title{
Even coefficient estimates for bounded univalent functions
}

\author{
by D. V. Prokhorov (Saratov)
}

\begin{abstract}
Extremal coefficient properties of Pick functions are proved. Even coefficients of analytic univalent functions $f$ with $|f(z)|<M,|z|<1$, are bounded by the corresponding coefficients of the Pick functions for large $M$. This proves a conjecture of Jakubowski. Moreover, it is shown that the Pick functions are not extremal for a similar problem for odd coefficients.
\end{abstract}

Let $S$ denote the class of functions $f$,

$$
f(z)=z+\sum_{n=2}^{\infty} a_{n} z^{n}
$$

analytic and univalent in the unit disk $E=\{z:|z|<1\}$. Let $S^{M}, M>1$, denote the family of functions $f \in S$ bounded by $M:|f(z)|<M$ for $|z|<1$. Moreover, set $S^{\infty}=S$.

L. de Branges [1] proved the Bieberbach conjecture: $\left|a_{n}\right| \leq n, n \geq 2$, in the class $S$, with equalities only for the Koebe functions $K_{\alpha}$,

$$
K_{\alpha}(z)=\frac{z}{\left(1-e^{i \alpha} z\right)^{2}}, \quad \alpha \in \mathbb{R} .
$$

The functions $P_{\alpha}^{M} \in S^{M}$ which satisfy the equation

$$
\frac{M^{2} P_{\alpha}^{M}(z)}{\left(M-P_{\alpha}^{M}(z)\right)^{2}}=K_{\alpha}(z), \quad|z|<1, M>1, \quad P_{\alpha}^{\infty}=K_{\alpha},
$$

are called Pick functions. Let

$$
P_{0}^{M}(z)=z+\sum_{n=2}^{\infty} p_{n, M} z^{n}, \quad 1<M \leq \infty, \quad p_{n, \infty}=n .
$$

1991 Mathematics Subject Classification: Primary 30C50.

Key words and phrases: coefficient estimates, univalent function, Pick function, Koebe function. 
Z. Jakubowski [4] conjectured that even coefficients of functions $f \in S^{M}$ are bounded by $p_{n, M}$ for large $M$. Namely, for every even $n \geq 2$ there exists $M_{n}^{+}>1$ such that for all $M \geq M_{n}^{+}$and all $f \in S^{M}$,

$$
\left|a_{n}\right| \leq p_{n, M} .
$$

For references to earlier results due to Z. Jakubowski, A. Zielińska, K. Zyskowska, L. Pietrasik, M. Schiffer, O. Tammi, O. Jokinen, see [4]. Recently the author's student V. G. Gordenko [3] proved the Jakubowski conjecture for $n=6$. Moreover, he showed that Pick functions do not maximize $\left|a_{5}\right|$ in $S^{M}$ with finite $M$.

In this article we prove the Jakubowski conjecture for all even $n \geq 2$. Moreover, we show that odd coefficients of functions $f \in S^{M}$ do not necessarily satisfy (2) for sufficiently large $M$.

1. According to [1] only Koebe functions are extremal for the estimate of $\left|a_{n}\right|$ in $S$. Since the classes $S^{M}$ are rotation invariant, it is sufficient to find an upper estimate for Re $a_{n}$ instead of one for $\left|a_{n}\right|$. Thus the Jakubowski conjecture reduces to the fact that only Pick functions $P_{0}^{M}$ and their rotations give a local maximum of $\operatorname{Re} a_{n}$ in the class $S^{M}$ for large $M$.

The author [6], [7] described a constructive algorithm determining the value set $V_{n}^{M}$ of the coefficient system $\left\{a_{2}, \ldots, a_{n}\right\}$ in the class $S^{M}, 1<M$ $\leq \infty$. The set $V_{n}^{M}$ is the set reachable at time $t=\log M$ for the dynamical control system

$$
\frac{d a}{d t}=-2 \sum_{s=1}^{n-1} e^{-s(t+i u)} A(t)^{s} a(t), \quad a(0)=a^{0},
$$

where $a=a(t) \in \mathbb{C}^{n}$,

$$
a(t)=\left(\begin{array}{c}
a_{1}(t) \\
\vdots \\
a_{n}(t)
\end{array}\right), \quad A(t)=\left(\begin{array}{ccccc}
0 & 0 & \ldots & 0 & 0 \\
a_{1}(t) & 0 & \ldots & 0 & 0 \\
a_{2}(t) & a_{1}(t) & \ldots & 0 & 0 \\
\ldots \ldots \ldots \ldots \ldots \ldots \ldots \ldots \ldots \ldots . \\
a_{n-1}(t) & a_{n-2}(t) & \ldots & a_{1}(t) & 0
\end{array}\right),
$$

$a^{0}=(1,0, \ldots, 0)^{T}, a_{1}(t) \equiv 1$, and $u=u(t)$ is a real control. Optimal controls satisfy the Pontryagin maximum principle. They maximize the Hamilton function

$$
H(t, a, \bar{\psi}, u)=-2 \sum_{s=1}^{n-1} \operatorname{Re}\left[e^{-s(t+i u)}\left(A^{s} a\right)^{T} \bar{\psi}\right]
$$

while the conjugate vector $\bar{\psi}=\left(\bar{\psi}_{1}, \ldots, \bar{\psi}_{n}\right)^{T}$ of complex-valued Lagrange 
multipliers satisfies the conjugate Hamilton system

$$
\frac{d \bar{\psi}}{d t}=2 \sum_{s=1}^{n-1} e^{-s(t+i u)}(s+1)\left(A^{T}\right)^{s} \bar{\psi}, \quad \psi(0)=\xi .
$$

The vector $\left(\psi_{2}(\log M), \ldots, \psi_{n}(\log M)\right)$ is orthogonal to the boundary hypersurface $\partial V_{n}^{M}$ of $V_{n}^{M}$. More precisely, it is orthogonal to a tangent plane or to a certain support plane if they exist. If $\operatorname{Re} a_{n}$ attains its maximum at any point $x \in \partial V_{n}^{M}$, then there exists $\psi$ such that $\left(\psi_{2}(\log M), \ldots, \psi_{n}(\log M)\right)=$ $(0, \ldots, 0,1)$ at this point.

Points of $\partial V_{n}^{M}$ are obtained from boundary extremal functions $f, f(z)=$ $M w(z, \log M)$, where $w(z, t)$ are solutions of the Cauchy problem for Loewner's differential equation

$$
\frac{d w}{d t}=-w \frac{e^{i u}+w}{e^{i u}-w},\left.\quad w\right|_{t=0}=z,
$$

with optimal controls $u=u(t)$. Differentiating (5) with respect to $z$, we obtain a differential equation for $w^{\prime}(z, t)$, from which we deduce differential equations for the coefficient system $b(t)=\left\{b_{0}(t), \ldots, b_{n-1}(t)\right\}$ of the function $f^{\prime}(z) /\left(e^{t} w^{\prime}(z, t)\right)$. The system for $b(t)$ coincides with (4) with $A^{T}$ replaced by $A$. Hence if $\left(\psi_{2}(\log M), \ldots, \psi_{n}(\log M)\right)=(0, \ldots, 0,1)$, then

$$
\left(\psi_{2}(t), \ldots, \psi_{n}(t)\right)=\left(b_{n-2}(t), \ldots, b_{0}(t)\right) .
$$

The initial value at $t=0$ yields that $\xi=\left(\xi_{1},(n-1) a_{n-1}, \ldots, 2 a_{2}, 1\right)^{T}$.

2. Now we are able to prove the theorem for odd coefficients of $f \in S^{M}$.

THEOREM 1. The Pick functions $P_{0}^{M}$ are not extremal for the problem of estimating Re $a_{2 m+1}$ in the class $S^{M}$, for all sufficiently large finite $M$ and natural $\mathrm{m}$.

Proof. $P_{0}^{M}$ and $K_{0}$ correspond to the control $u(t) \equiv \pi$ in $(3)-(4)$. In this case the condition $\left(\psi_{2}(\log M), \ldots, \psi_{n}(\log M)\right)=(0, \ldots, 0,1)$ requires the initial value $\left(\xi_{2}, \ldots, \xi_{n}\right)=\left((n-1) p_{n-1, M}, \ldots, 2 p_{2, M}, 1\right), 1<M \leq \infty$, in (4).

Put $n=2 m+1$ and write the Hamilton function at $t=0$,

$$
H\left(0, a^{0}, \bar{\xi}, u\right)=-2 \sum_{s=1}^{2 m} \xi_{s+1} \cos (s u) \text {. }
$$

Hence

$$
\frac{\partial H\left(0, a^{0}, \bar{\xi}, u\right)}{\partial u}=2 \sum_{s=1}^{2 m} s \xi_{s+1} \sin (s u)
$$


and this derivative vanishes at $u=\pi$. Moreover,

$$
\left.\frac{\partial^{2} H\left(0, a^{0}, \bar{\xi}, u\right)}{\partial u^{2}}\right|_{u=\pi}=2 \sum_{s=1}^{2 m}(-1)^{s} s^{2} \xi_{s+1} .
$$

Evidently this derivative vanishes if $M=\infty$. It must be non-positive for finite $M$ if $u \equiv \pi$ satisfies Pontryagin's maximum principle.

Let us examine how this derivative depends on $M$. Write

$h(M)=\sum_{s=1}^{2 m}(-1)^{s} s^{2} \xi_{s+1}=\sum_{s=1}^{2 m}(-1)^{s} s^{2}(2 m+1-s) p_{2 m+1-s, M}, \quad p_{1, M}=1$.

Every coefficient $p_{j, M}$ can be found from (3). It is the $j$ th coordinate of the vector $a(\log M)$ if $u(t) \equiv \pi$. Put $T=1-1 / M, h(M)=h(1 /(1-T))=g(T)$. Then by elementary calculations we find from (3) that

$$
\left.\frac{d g}{d T}\right|_{T=1}=\frac{1}{3} \sum_{s=1}^{2 m-1}(-1)^{s} s^{2}(2 m-s)(2 m+1-s)^{2}(2 m+2-s) .
$$

One can verify that $(1 / 12)(j+1)(j+2)^{2}(j+3)$ is the $j$ th coefficient of the function $(1-z)^{-4}+2 z(1-z)^{-5}$ while $(-1)^{s} s^{2}$ is the $(s-1)$ th coefficient of $(z-1)(z+1)^{-3}$. Thus $\left.\left(-\frac{1}{4}\right) \frac{d g}{d T}\right|_{T=1}$ is the $(2 m-2)$ th coefficient of $\left(1-z^{2}\right)^{-2}(1-z)^{-2}$, and it is positive. Hence $h(M)$ is decreasing for sufficiently large $M$. Since $h(\infty)=0$, we conclude that $h(M)>0$ for large $M$.

The last result contradicts the maximizing property of the control $u=\pi$. This proves Theorem 1.

3. Now we are going to investigate the extremal properties of even coefficients of Pick functions.

THEOREM 2. For every natural $m$ there exists $M_{2 m}^{+}>1$ such that each function $f \in S^{M}$ satisfies the inequalities (2) for $n=2 m$ and all $M \geq M_{2 m}^{+}$.

Proof. Let $X$ denote an arbitrary neighbourhood of the function $K_{0}$ in the class $S$, endowed with the topology of uniform convergence on compact subsets of the unit disk. Set $X^{M}=X \cap S^{M}$. The Pick function $P_{0}^{M}$ belongs to $X^{M}$ for sufficiently large $M$. By Section 1, it is sufficient to show that only $P_{0}^{M}$ gives a local maximum for $\operatorname{Re} a_{n}$ in $X^{M}$.

Again we have $\left(\psi_{2}(\log M), \ldots, \psi_{n}(\log M)\right)=(0, \ldots, 0,1)$ at a point $x \in \partial V_{n}^{M}$ where $\operatorname{Re} a_{n}$ attains its maximum. If $x$ comes from a function $f \in S^{M}$ with expansion (1), then we need the initial value $\left(\xi_{2}, \ldots, \xi_{n}\right)=$ $\left((n-1) a_{n-1}, \ldots, 2 a_{2}, 1\right)$ in (4).

Put $n=2 m, \xi^{0}=\left(\xi_{1},(2 m-1)^{2}, \ldots, 1\right)^{T}$. Then

$$
H\left(0, a^{0}, \bar{\xi}^{0}, u\right)=-2 \sum_{s=1}^{2 m-1}(2 m-s)^{2} \cos (s u) .
$$


By elementary calculations we find that

$$
H\left(0, a^{0}, \bar{\xi}^{0}, u\right)-H\left(0, a^{0}, \bar{\xi}^{0}, \pi\right)=\frac{(-\sin u)[2 m \sin u-\sin (2 m u)]}{(1-\cos u)^{2}} .
$$

It is easy to verify that the right-hand side of this equality is negative on $[0,2 \pi]$, except for $u=\pi$, where it vanishes. Thus

$$
H\left(0, a^{0}, \bar{\xi}^{0}, u\right) \leq H\left(0, a^{0}, \bar{\xi}^{0}, \pi\right),
$$

with equality only for $u=\pi$. Moreover,

$$
\frac{\partial H\left(0, a^{0}, \bar{\xi}^{0}, u\right)}{\partial(\cos u)}=2 \sum_{s=1}^{2 m-1}(-1)^{s} s^{2}(2 m-s)^{2} .
$$

This is the $(2 m-2)$ th coefficient of $-2\left(1-z^{2}\right)^{-2}$, and it is negative.

The sign of this derivative and the inequality (7) are preserved for close points $\xi$. Let $\xi=\left(\xi_{1}, \ldots, \xi_{n}\right)^{T}$ be an arbitrary point in a neighbourhood of $\xi^{0}$, with $\xi_{2}, \ldots, \xi_{n}$ real. Then according to the continuity principle $H\left(0, a^{0}, \bar{\xi}, u\right)$ attains its maximum on $[0,2 \pi]$ at the single point $u=\pi$. We can choose $\left(\xi_{2}, \ldots, \xi_{n}\right)=\left((n-1) p_{n-1, M}, \ldots, 2 p_{2, M}, 1\right)$ for sufficiently large $M$. The control $u=\pi$ satisfies Pontryagin's maximum principle for $t>0$ in a certain neighbourhood of the initial value $t=0$, and the corresponding solution $w(z, t)$ of Loewner's differential equation (5) has real coefficients. Hence $u=\pi$ is optimal on the whole half-axis $[0, \infty)$ (see e.g. [6], [7]). This gives the Pick function $P_{0}^{M}$. So $P_{0}^{M}$ satisfies the necessary conditions for maximum of $\operatorname{Re} a_{n}$.

It remains to show that the necessary conditions for an extremum hold at a unique point in $X^{M}$.

Let us consider the point $a=(1,2, \ldots, n)^{T}$ in $\partial V_{n}=\partial V_{n}^{\infty}$ and its neighbourhood $Q_{a}, Q_{a} \subset \partial V_{n}$. Points of $Q_{a}$ appear as the phase space projections of solutions of the Cauchy problem for the Hamilton system (3), (4). The neighbourhood $Q_{a}$ corresponds to a neighbourhood $Q_{\xi}$ of the initial value $\Lambda=\left(\xi_{2}, \ldots, \xi_{n}\right)=\left((n-1)^{2}, \ldots, 1\right)$ in $(4)$. This correspondence is not one-to-one. All points $\xi^{*} \in Q_{\xi}$ with real coordinates $\xi_{2}^{*}, \ldots, \xi_{n}^{*}$ are mapped to the point $a$. The correspondence between the conjugate vector and the initial value is one-to-one in $Q_{\xi}$. This means that the hypersurface $\partial V_{n}$ does not have any tangent hyperplane at $a$. It has support hyperplanes there. The initial value $\Lambda$ selects the support hyperplane $\Pi$ with normal vector $(0, \ldots, 0,1)$. But $\Pi$ and $\partial V_{n}$ may be tangent along some directions in the imaginary parts of coordinates of the phase vector, i.e. along the directions of the imaginary parts of $\xi_{2}, \ldots, \xi_{n}$. We will show that this is at most first order tangency. 
Let $(a(t), \psi(t))$ solve the Cauchy problem (3)-(4) with $u=\pi$ and with initial value $\Lambda$, and let $\Lambda^{*}=\left(\xi_{2}^{*}, \ldots, \xi_{n}^{*}\right)=\Lambda+\varepsilon\left(\delta_{2}, \ldots, \delta_{n}\right)$, where $\varepsilon>0$, and $\delta_{2}, \ldots, \delta_{n}$ are constant complex numbers. Suppose that $\Pi$ and $\partial V_{n}$ have second order tangency along the direction determined by $\left(\delta_{2}, \ldots, \delta_{n}\right)$. The phase vector $a^{*}(t)$ and the conjugate vector $\psi^{*}(t)$ solve the Cauchy problem $(3)-(4)$ with $\psi^{*}(0)=\left(\xi_{1}, \xi_{2}^{*}, \ldots, \xi_{n}^{*}\right)^{T}$ and with optimal control $u^{*}=u^{*}\left(t, a^{*}, \bar{\psi}^{*}\right)$.

Second order tangency implies that $\operatorname{Re} a_{n}^{*}(\infty)=n+O\left(\varepsilon^{3}\right)$. Since $\left|a_{n}^{*}(\infty)\right|$ $\leq n$, we have $\operatorname{Im} a_{n}^{*}(\infty)=O\left(\varepsilon^{2}\right)$, and so $a_{n}^{*}(\infty)=n+O\left(\varepsilon^{2}\right)$. By E. Bombieri's result stated in [5], there are constants $\alpha_{n}$ and $\beta_{n}$ such that $\operatorname{Re}\left(2-a_{2}\right)$ $<\alpha_{n} \operatorname{Re}\left(n-a_{n}\right)$ for $n$ even, and $\left|2-a_{2}\right| \leq \beta_{n}$. It follows that $\operatorname{Re} a_{2}^{*}(\infty)=$ $2+O\left(\varepsilon^{3}\right), \operatorname{Im} a_{2}^{*}(\infty)=O\left(\varepsilon^{2}\right)$, and so $a_{2}^{*}(\infty)=2+O\left(\varepsilon^{2}\right)$. By D. Bshouty's result [2], there exist constants $c_{k}$ and $d_{k}$ such that for $k \geq 2, \operatorname{Re}\left(k-a_{k}\right)$ $\leq c_{k} \operatorname{Re}\left(2-a_{2}\right)$ and $k-\left|a_{k}\right| \leq d_{k} \operatorname{Re}\left(2-a_{2}\right)$. It follows that for $2 \leq$ $k \leq n, \operatorname{Re} a_{k}^{*}(\infty)=k+O\left(\varepsilon^{3}\right), \operatorname{Im} a_{k}^{*}(\infty)=O\left(\varepsilon^{2}\right)$, and so $a_{k}^{*}(\infty)=$ $k+O\left(\varepsilon^{2}\right)$. Hence $\left(\psi_{2}^{*}(\infty), \ldots, \psi_{n}^{*}(\infty)\right)=(0, \ldots, 0,1)+O(\varepsilon)$. The relation (6) at $t=0$ implies that $\Lambda^{*}=\Lambda+O\left(\varepsilon^{2}\right)$. This contradicts our assumptions.

Thus the hyperplane $\Pi$ may have at most first order tangency to $\partial V_{n}$ along some directions. $\Pi$ is the unique support hyperplane with normal vector $(0, \ldots, 0,1)$ in the neighbourhood $Q_{a}$. The hypersurfaces $\partial V_{n}^{M}$ depend analytically on $M$, except for manifolds of smaller dimension. Hence, passing from $\partial V_{n}$ to $\partial V_{n}^{M}$, we have the unique support hyperplane with normal vector $(0, \ldots, 0,1)$ in a neighbourhood $Q_{a}^{M} \subset \partial V_{n}^{M}$ of the point $a^{M}=\left(1, p_{2, M}, \ldots, p_{n, M}\right)^{T}$, for $M$ sufficiently large. This ends the proof.

Theorem 2 answers affirmatively the Jakubowski conjecture.

\section{References}

[1] L. de Branges, A proof of the Bieberbach conjecture, Acta Math. 154 (1985), $137-152$.

[2] D. Bshouty, A coefficient problem of Bombieri concerning univalent functions, Proc. Amer. Math. Soc. 91 (1984), 383-388.

[3] V. G. Gordenko, Sixth coefficient estimate for bounded univalent functions, in: Theory of Functions and Approximation, Proc. 6th Saratov Winter School, Saratov (in Russian), to appear.

[4] Z. Jakubowski, On some extremal problems in classes of bounded univalent functions, Zeszyty Nauk. Politechn. Rzeszowskiej Mat. Fiz. 16 (2) (1984), 9-16 (in Polish).

[5] C. Pommerenke, Univalent Functions, Vandenhoeck and Ruprecht, Göttingen, 1975. 
[6] D. V. Prokhorov, Value sets of systems of functionals in classes of univalent functions, Mat. Sb. 181 (12) (1990), 1659-1677 (in Russian).

[7] - Reachable Set Methods in Extremal Problems for Univalent Functions, Izdat. Saratov. Univ., 1992.

FACULTY OF MATHEMATICS AND MECHANICS

SARATOV STATE UNIVERSITY

ASTRAKHANSKAYA, 83

410071 SARATOV, RUSSIA

Reçu par la Rédaction le 7.7.1992 